\section{Ressecção Parcial de Palato (RPP) paratratamento de roncopatia pela Técnica de Quesada e Perelló: resultados}

\author{
Luis F. Oliveira', Ana C. P. B. Soter ${ }^{1}$, \\ Flávio L.P. Silva ${ }^{1}$, Ângelo U. Panerari ${ }^{1}$, \\ Floriano P. Rocha J $r^{2}$, Antonio C. Cedin ${ }^{3}$
}

\section{Partial Resection of the Palate in the treatment of snoring by Quesada \& Perelló technique: results}

Resumo / Summary

$\mathbf{R}$ sente em $38 \%$ das mulheres e $40 \%$ dos homens, com importantes implicações sociais. Várias técnicas são descritas na literatura para a correção da hipertrofia do palato mole, associadas a outros procedimentos, com o propósito de remissão dos sintomas. Objetivo: Para estudar os resultados da técnica proposta por Quesada e Perelló, denominada Ressecção Parcial do Palato (RPP), avaliamos a remissão do ronco, as complicações e o conforto pós-operatório do paciente. Forma de estudo: Clínico retrospectivo. Material e Método: 28 pacientes foram arrolados no estudo, de abril de 1994 a agosto de 2000, mas apenas 23 preencheram os critérios de inclusão, com queixas de ronco sem alterações compatíveis com apnéia obstrutiva. Todos os pacientes apresentavam classificação de Fujita tipo I ou II e foram avaliados com polissonografia, tomografia computadorizada e exame otorrinolaringológico completo. Resultados: Após 6 meses, a remissão dos sintomas foi total em 12 casos (42\%), parcial em 9 (32\%) e inalterado em $2(7 \%) ; 5$ pacientes não completaram o acompanhamento de seis meses. Conclusão: A técnica de Ressecção Parcial do Palato proposta por Quesada et al. e Perelló mostrou-se segura e eficaz no tratamento do ronco.
Palavras-chave: ronco, uvulopalatoplastia, cirurgia, síndrome da apnéia obstrutiva do sono. Key words: snoring, uvulopalatalplasty, surgery, sleep apnea obstructive syndrome.
Snoring is a relatively common clinical entity, present in $38 \%$ of women and $40 \%$ of men, with important social implications. Various techniques are described in the literature for the correction of hypertrophy of the soft palate, besides other associated procedures for the purpose of symptom remission. Aim: in order to study the results of the technique proposed by Quesada e Perelló, which was denominated Partial Resection of the Palate, we evaluated the remission of the snoring, its complications and post-operative comfort of the patient. Study design: Clinical retrospective. Material and Method: 28 patients were submitted to surgery of the palate from April 1994 to August 2000 with the complaint of snoring without clinical alterations compatible with obstructive apnea. All patients presented the type I or type Il of Fujita classification and were evaluated with polysonography, computerized tomography and complete ear, nose and throat exam. Results: after 6 months, remission of symptom was complete in 12 patients (52\%), partial in 9 $(39 \%)$ and unchanged in $2(9 \%) ; 5$ patients did not complete the study at 6 months. Conclusion: the partial resection technique proposed by Quesada et al. and Perelló proved to be safe and efficient in the treatment of snoring.

\footnotetext{
${ }^{1}$ Residentes de Otorrinolaringologia.

2 Preceptor da Residência.

${ }^{3}$ Coordenador da Residência.

Serviço de O torrinolaringologia do Hospital Beneficência Portuguesa - Clínica Ivan F. Barbosa.

Endereço para Correspondência: Luis Francisco de Oliveira - R. Maestro Cardim, 770 Bela Vista São Paulo SP 01320-001. Tel (0xx11)288-0899 - Fax (0xx11)3253-0705 - E-mail: If_otorrino@uol.com.br

Trabalho apresentado no 36 Congresso Brasileiro de Otorrinolaringologia - Florianópolis/SC, novembro de 2002, como tema livre, no dia 22/11/2002, sala 12. Artigo recebido em 03 de junho de 2003. Artigo aceito em 15 de janeiro de 2004.
} 


\section{INTRODUÇÃO}

0 ronco é o som emitido, durante o sono, pela vibração das partes moles da faringe (úvula, palato, pilares amigdalianos, base da língua) durante a passagem de ar e ocorre devido ao aumento da resistência à permeabilidade do fluxo de ar pelas vias aéreas superiores ${ }^{1}$. Acomete adultos e crianças e pode acarretar problemas sociais importantes; quando associado a despertares freqüentes e sonolência diurna, com achados polissonográficos normais, caracteriza a Síndrome da Resistência das Vias Aéreas Superiores ${ }^{2} e$ quando está relacionado à Síndrome da Apnéia-Hipopnéia Obstrutiva do Sono (SAHOS), pode acarretar outras comorbidades. É uma queixa dotada de muita subjetividade, pois pode sofrer influências emocionais de quem as relata. A informação vem do próprio paciente ou de seu companheiro, o que a torna super ou subestimada, a depender da qualidade do sono dos informantes ${ }^{3}$. Estudos na literatura mostram que quando se comparou a queixa de ronco relatada pelo paciente, pelo companheiro e por um terceiro informante não relacionado aos anteriores, houve discrepância de informações ${ }^{4}$. Ainda segundo a literatura, sua incidência pode variar de $28 \%$ na população adulta feminina a $44 \%$ nos homens ${ }^{5-8} \mathrm{e}$ esses valores tendem a aumentar com a idade até a 7a década de vida, podendo chegar a $84 \%$ em homens e $73 \%$ em mulheres nessa faixa etária 8 . Ainda que a ocorrência de ronco e apnéia sejam reconhecidas há muito tempo (existem relatos de 360 a.C.), foi nos últimos 30 anos que tomou corpo no mundo científico ${ }^{1}$. Diversos tratamentos clínicos e cirúrgicos foram propostos já a partir do início da década de 50 com grande divulgação a partir dos anos $80^{\circ}$. As bases para o tratamento, independente da técnica cirúrgica a ser utilizada, são as mesmas: ampliar a coluna aérea para os pulmões, diminuindo a resistência ao fluxo de ar, seja por remoção de tecidos moles (palato, pilares, língua), seja por deslocamentos da estrutura óssea (mandibular, maxilar). Entre as técnicas descritas estão a osteotomia com avanço maxilomandibular e suas derivações ${ }^{10}$, suspensão do hióide ${ }^{11}$, traqueostomia ${ }^{12,13}$, adenoidectomia e/ou amigdalectomia, cirurgia nasal, somnoplastia, laser de $\mathrm{CO}_{2}$ uvulopalatofaringoplastia (UPFP) ${ }^{9} \mathrm{e}$ a Ressecção Parcial de Palato (RPP) ${ }^{14}$, essa última o objeto de nosso estudo. 0 objetivo foi avaliar os resultados na remissão do ronco, bem como a dor pós-operatória e complicações, com a utilização da técnica de RPP descrita por Quesada et al. e Perelló ${ }^{14} \mathrm{em}$ pacientes com queixa de ronco.

\section{MATERIAL E MÉTODO}

Foi realizado estudo retrospectivo de 28 pacientes submetidos à Ressecção Parcial de Palato (RPP), no período de abril de 1994 a agosto de 2000; 5 pacientes apresentaram critérios de exclusão. A idade variou de 20 a 75 anos, com idade média de 46,7 , sendo que a maioria (17) se en- contrava entre 30 e 50 anos. Predominou o sexo masculino com 25 pacientes contra 3 do sexo feminino.

Todos os pacientes foram avaliados previamente pelos seguintes parâmetros: CT de fossas nasais e cavidades paranasais, polissonografia e exame otorrinolaringológico completo. Na polissonografia, os parâmetros considerados foram: Índice de Apnéia-Hipopnéia/hora (IAH), saturação mínima de oxigênio ( satO $_{2}$ ) e intensidade dos roncos. Define-se apnéia e hipopnéia como a cessação total ou parcial, respectivamente, do fluxo aéreo nasal/oral por mais de 10 segundos e, segundo a Academia Americana de Medicina do Sono (AASM), a SAHOS é caracterizada por episódios recorrentes de obstrução parcial ou total das vias aéreas superiores durante o sono $0^{15}$.

O IAH foi classificado como normal quando existiram até cinco eventos por hora, leve de 5 a 15 eventos, moderada de 15 a 30 e grave quando superava a marca dos trinta eventos por hora ${ }^{15}$. A intensidade do ronco foi classificada ${ }^{1}$ em:

Grau I: ronco ocasional, em decúbito dorsal, associado a cansaço excessivo, ingesta de álcool e/ ou alimentos

Grau II: ronco muito alto, freqüente, em qualquer posição, e que pode ser ouvido nos outros cômodos da casa próximos

Grau III: ronco excessivamente alto, que pode ser ouvido em lugar da casa, geralmente associado a SAHOS.

Quanto à saturação de oxigênio, classificou-se como saturação normal acima de $90 \%$, dessaturação leve de $85 \%$ a $89 \%$, moderada de $65 \%$ a $84 \%$ e intensa abaixo de $85 \%$.

No exame físico dos pacientes submetidos a essa técnica encontrou-se hipertrofia de palato mole e/ ou pilar posterior redundante em todos os pacientes; desvio de septo nasal em 18 casos (64\%), hipertrofia de cornetos inferiores em $3(11 \%)$ e hipertrofia de tonsila palatina/lingual em 1 (3,5\%). Os achados nasossinusais foram confirmados pela CT das cavidades paranasais.

A polissonografia revelou IAH leve/normal em 22 pacientes (78,5\%), moderada em $4(14 \%)$ e grave em 2 (7\%). A satO ${ }_{2}$ manteve-se normal em 19 casos (68\%), hipóxia leve em $6(21 \%)$ e grave em $3(11 \%)$. Roncos grau III foram observados em 7 casos (25\%), grau II em 5 casos (18\%) grau I em 16 (57\%).

Foram incluídos no estudo apenas os pacientes com IAH leve ou normal, IMC $<25$ sem sinais clínicos de privação importante do sono segundo a escala de Epworth e sem qualquer outra comorbidade; foram excluídos da avaliação dos 6 meses 5 pacientes que apresentavam alguma comorbidade, sendo $2 \mathrm{com}$ IAH grave e $3 \mathrm{com} \mathrm{IAH} \mathrm{mode-}$ rada. Apenas 1 paciente com IAH moderada foi incluído por não apresentar, clinicamente, sintomas de sonolência diurna excessiva. 


\section{Técnica}

1. Marcação do limite do palato duro (com bisturi elétrico) como ponto de referência; amigdalectomia, quando presente

2. Ressecção parcial da úvula (Figura 1)

3. Eversão do coto de úvula para marcação dos limites do descolamento mucoso de modo a restar entre $1,5 \mathrm{~cm}$ e $2,0 \mathrm{~cm}$ de comprimento no palato mole e lateralmente até $1,0 \mathrm{~cm}$ do último molar superior

4. Descolamento com bisturi elétrico de mucosa e submucosa da face oral do palato (Figura 2)

5. Eversão e sutura com vicryl 3.0 em pontos separados (Figura 3), com aspecto final sem área cruenta e sem ressecção de musculatura do palato (Figura 4).

Foram avaliados, no pós-operatório, a dor, medida através de relatos dos pacientes, necessidade do uso de analgésicos e reintrodução da dieta normal; complicações imediatas (hemorragia, hematoma, edema obstrutivo de vias aéreas) e tardias (insuficiência velofaríngea, mudança de voz, estenose, mioclonia, perda do paladar, infecção bacteriana, refluxo nasal de alimentos) ${ }^{16}$ e remissão do ronco. Esses dados foram avaliados de forma subjetiva através das informações prestadas pelo paciente e seus familiares. Não se realizou polissonografia no seguimento de seis meses porque o objetivo do estudo foi avaliar apenas a resolução da roncopatia.

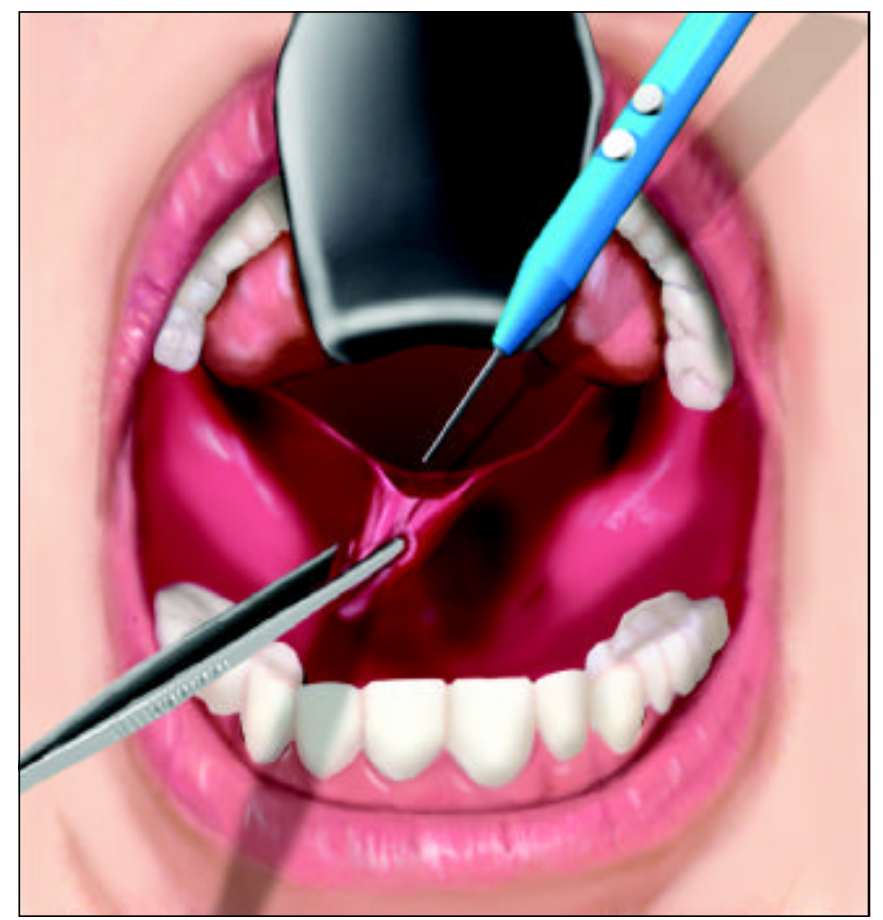

Figura 1. Ressecção parcial da úvula, na altura em que será suturada na superfície anterior do palato

\section{RESULTADOS}

A remissão da dor com suspensão total de analgésicos ocorreu em uma semana para 6 pacientes (21\%), 2 semanas para 19 pacientes (68\%) e 3 semanas para os outros três pacientes (11\%). Com relação à introdução de dieta geral, todos foram orientados a manter dieta líquida e/ ou pastosa na primeira semana para diminuir riscos de complicações e, após esse período, foram liberados para dieta normal. Ao término da $2^{a}$ semana, todos já haviam retomado a dieta habitual.

A melhora do ronco, após seis meses, foi total em 12 casos (42\%), parcial em 9 (32\%) e inalterado em $2(7 \%)$, com cinco pacientes não completando o estudo.

Apenas um paciente apresentou rinolalia transitória como complicação pós-operatória e nenhum apresentou outra complicação até o sexto mês de pós-operatório. Nenhum paciente foi reoperado.

\section{DISCUSSÃO}

A terapêutica cirúrgica para o ronco consiste no tratamento da hipertrofia do palato, amígdalas e cirurgia nasal. Entre as técnicas descritas na literatura para o palato, destacam-se a cirurgia com laser de $\mathrm{CO}_{2}$ a somnoplastia, a uvulopalatofaringoplastia e a ressecção parcial de palato (RPP). A abordagem do palato para tratamento da roncopatia

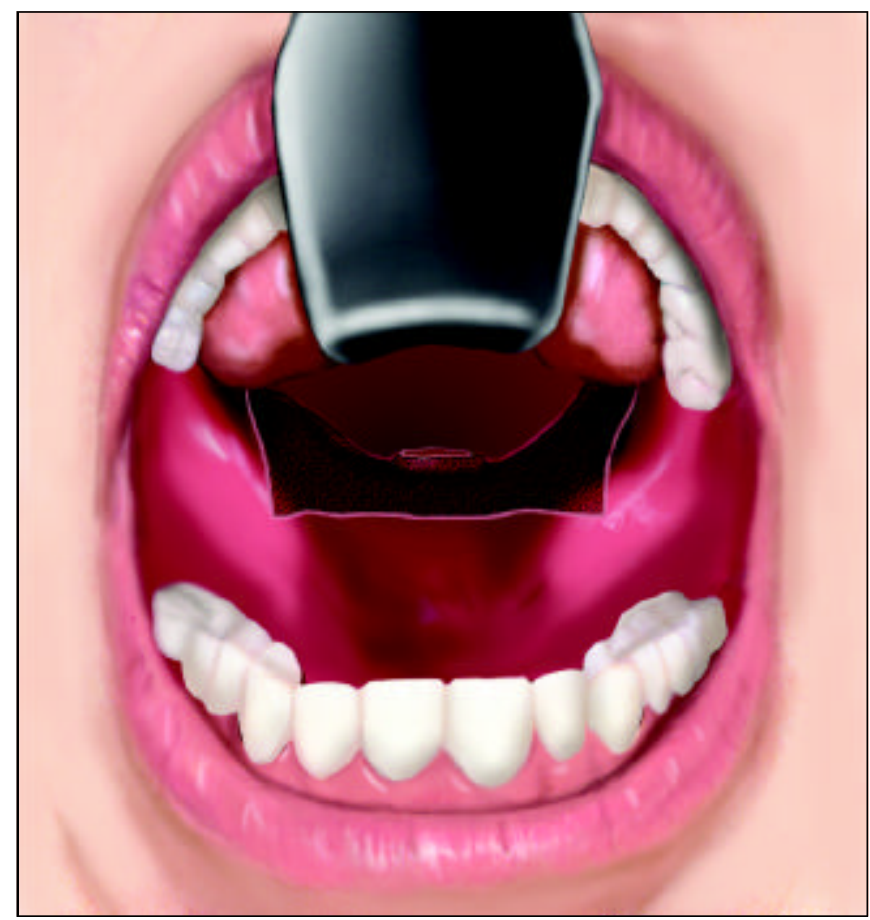

Figura 2. Mucosa anterior do palato mole ressecada, sem perda de tecido muscular 


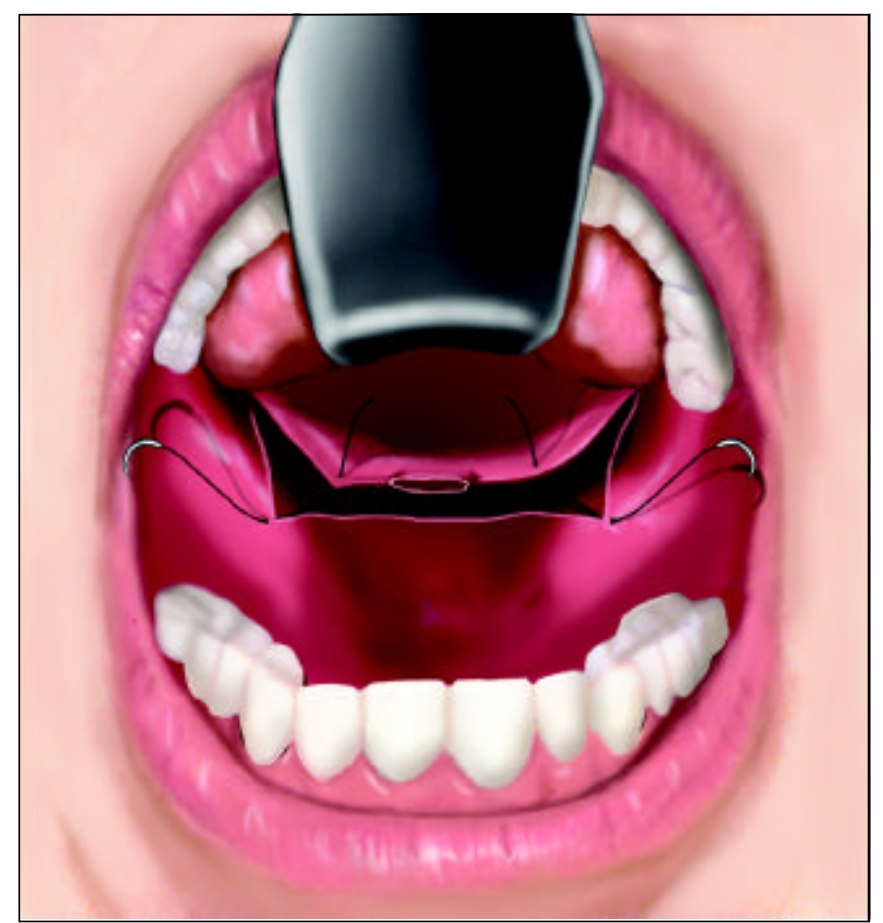

Figura 3. Eversão e sutura

foi descrita, inicialmente, na década de 50 e introduzida nos EUA nos anos $80^{\circ}$. 0 ronco isolado, normalmente, não acarreta grandes repercussões sistêmicas, mas a apnéia pode implicar em comprometimento importante na qualidade de vida (cansaço, sonolência diurna, irritabilidade, alterações de humor e cognição) ${ }^{1}$, de ordem geral (HAS, arritmia, IAM, ICC, morte súbita noturna) ${ }^{1}$ e risco aumentado para acidentes de trânsito ${ }^{17}$, merecendo uma atenção especial. Em crianças as repercussões também se manifestam no crescimento pôndero-estatural prejudicado, crescimento do terço médio da face e prejuízo das atividades escolares.

No presente estudo considerou-se apenas a queixa de ronco dos pacientes, objetivando sua resolução. Estudou-se uma das técnicas cirúrgicas conhecidas, num seguimento de curto prazo ( 6 meses), período em que nosso estudo reproduziu os resultados encontrados na literatura mundial. Em alguns estudos mais longos mostrou-se que a taxa de sucesso da UPFP declina após algum tempo mantendo-se, então, estável. Levin e Becker ${ }^{18}$, avaliaram 69 pacientes com mais de 5 anos de seguimento e observaram que, após 13 meses a taxa de remissão total ou parcial do ronco reduziu de $87 \%$ para $46 \%$. Estudos com as outras técnicas de cirurgia do palato não mostraram resultados superiores aos aqui apresentados, não havendo, portanto, uma técnica que apresente sucesso total. Os defensores de cada técnica apresentam as

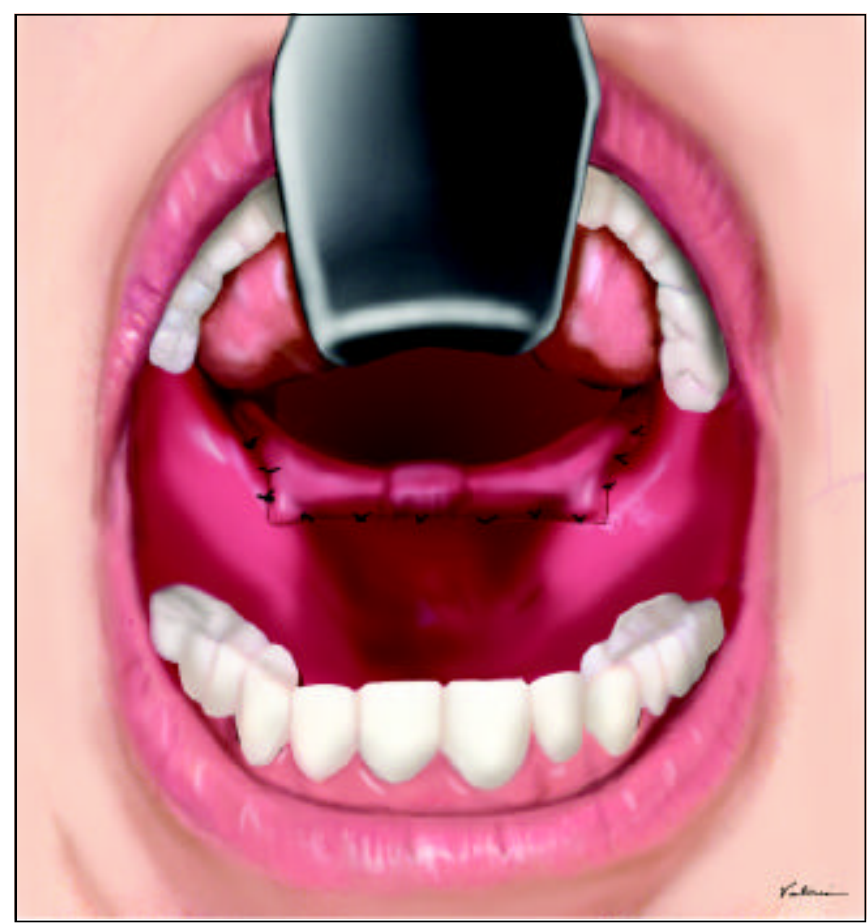

Figura 4. Aspecto final

potenciais vantagens de cada procedimento, não havendo, portanto, consenso na literatura mundial.

\section{CONCLUSÃO}

0 estudo acompanhou, por seis meses, 23 pacientes submetidos à cirurgia de ressecção parcial do palato para roncopatia, através da técnica proposta por Quesada e Perelló. Apresentou resultados satisfatórios na remissão do ronco, baixo risco quanto às complicações intra e pós-operatórias e melhora total da dor em duas semanas para a maioria dos pacientes, constituindo-se, portanto, numa boa alternativa para esse tipo de afecção.

\section{REFERÊNCIASBIBUOGRÁFICAS}

1. Pontes PAL. Ronco e Síndrome da Apnéia Obstrutiva do Sono (SAOS). In: Stamm A. ed. Rinologia 2000; São Paulo: Revinter; 2000:315-319

2. Guilleminaut $C$, Stools $R$, Clerk $A$ et al. From obstructive sleep apnea syndrome to upper airway resistance syndrome: consistency of day time sleepiness. Sleep 1992; 15:S13-S16.

3. Wiggins CL, Schmidt-Nowara WW, Coultas DB, Samet J. Comparison of self- and spouse reports of snoring and other symptoms associated with sleep apnea syndrome. Sleep 1991; 13:245-52.

4. Hoffstein V, Mateikas S, Anderson D. Snoring: is it in the ear of the beholder? Sleep 1994:17:522-6. 
5. Lugaresi E, Cirignotta F, Montagna P, Sforza E. Snoring: pathogenic, clinical, and therapeutic aspects. In: Kryger MH, Roth T, Dement WC, eds. Principles and practice of sleep medicine. Philadelphia: WB Saunders; 1994: 621-9.

6. Lugaresi E, Cirignotta F, Coccagna G, Piana C. Some epidemilogical data on snoring and cardiocirculatory disturbances. Sleep 1980; 3:221-4.

7. Young T, Palta M, Demsey J, Skatrud J, Weber S, Badr S. The occurrence of sleep-disordered breathing among middle-aged adults. N Engl J Med 1993; 328:1230-5.

8. Norton PG, Dun EV. Snoring as a risk factor for disease: an epidemilogical survey. BMJ 1984; 291:630-2.

9. Ikematsu T, Fujita S, Simmons FB, et al. Uvulopalatopharyngoplasty: variations in snoring and sleep obstrutive apnea. In: Fairbanks DNF, Fujita S, eds. Snoring and sleep obstrutive apnea, $2^{\text {nd }}$ ed. New York: Raven Press; 1994:97-146.

10. Riley RW, Powell NB, Guilleminaut C. Obstrutive sleep apnea syndrome: a review of 306 consecutively treated surgical pacients. Otolaryngol Head Neck Surg 1993; 108:117-25.
11. Riley RW, Powell NB, Guilleminaut C. Obstrutive sleep apnea and the hyoid: a revised surgical procedure. Otolaryngol Head Neck Surg 1994; 111:717-21.

12. Miller FR, Eliacgar I, Tucker HM. Technique, management, and complications of the long-term flap tracheostomy. Laryngoscope 1995; 105:543-7.

13. Sahni R, Blakeley B, Maisel RH. Flap tracheostomy in sleep apnea patients. Laryngoscope 1985; 95:221-3.

14. Quesada P, Pedro Botet J, Fuentes E, Perelló E. Resección parcial del paladar blando como tratamiento del sindrome de hipersomnia y respiración peródica de los obesos. ORL Dips 1977; 5:81-8.

15. AASM Task Force. Sleep 1999; 22(5): 667-89.

16. Pinto JA, Colombini NEP, Faller GJ. Complicações em cirurgias para apnéia obstrutiva do sono. In: Stamm A. ed. Rhinology 2002; São Paulo: Komedi; 2002:121-31.

17. Phillipson EA. Sleep apnea: a major public health problem. N Engl J Med 1993; 382:1271-3.

18. Levin BC, Becker GD. Uvulopalatopharyngoplasty for snoring: long-term results. Laringoscope 1994; 104:1150-2. 